\title{
ON PURITY AND RELATED UNIVERSAL PROPERTIES OF EXTENSIONS OF COMMUTATIVE RINGS
}

\author{
DAVID E. DOBBS
}

\begin{abstract}
Let $R \subseteq S$ be a unital extension of commutative rings. Then $R$ is a pure $R$-submodule of $S$ if and only if, for each finite set of algebraically independent indeterminates $\left\{X_{1}, \ldots, X_{n}\right\}$ over $S$ and each ideal $I$ of $R\left[X_{1}, \ldots, X_{n}\right]$, one has $I S\left[X_{1}, \ldots, X_{n}\right] \cap R\left[X_{1}, \ldots, X_{n}\right]=I$. Suppose also that $R$ is a Prüfer domain. Then $R$ is a pure $R$-submodule of $S$ if and only if, for each unital homomorphism of commutative rings $R \rightarrow T$, each chain of prime ideals of $T$ can be covered by a corresponding chain of prime ideals of $T \otimes_{R} S$.
\end{abstract}

\section{Introduction}

All rings considered below are commutative with identity; all ring homomorphisms, inclusions of rings and modules are unital. It will be convenient to say that a ring homomorphism $f: R \rightarrow S$ is contracted if each ideal $I$ of $R$ is contracted from $S$, in the sense that $I=f^{-1}(J)$ for some ideal $J$ of $S$ (equivalently, that $I=f^{-1}(I S)$ ). It is easy to see that any contracted ring homomorphism must be an injection (Lemma 1 (a)), and so we will focus mostly on inclusion maps of rings $R \hookrightarrow S$. A relevant moduletheoretic property is that of purity. Recall from [5, page 380] that if $R$ is a ring and $M$ is an $R$-submodule of an $R$-module $N$, then $M \hookrightarrow N$ is said to be pure if the canonical homomorphism $M \otimes_{R} E \rightarrow N \otimes_{R} E$ is an injection for each $R$-module $E$. It is well known that purity is somewhat of a universal property, inasmuch as it is preserved by flat base changes: see Lemma 1 (b) for a careful statement of this fact. A key result of Gilmer and Mott [12, page 752] states that if $R \subseteq S$ are rings such that $R \hookrightarrow S$ is pure (when viewed as an inclusion of $R$-modules), then $R \hookrightarrow S$ is contracted. The converse is false, as an example suggested by E. L. Lady in [4, Example 1] constructs an integral ring extension $R \subseteq S$ of Noetherian rings such that $R \hookrightarrow S$ is contracted but not pure (as $R$-modules). However, purity can be recovered from a universalized strengthening of the "contracted" property, as we next explain. By using the universality aspect of purity that was noted above, one sees that if $R \subseteq S$ are rings such that $R \hookrightarrow S$ is pure

Received June 13, 2009.

2000 Mathematics Subject Classification. Primary 13B99; Secondary 13A15, 13F05, 13C11.

Key words and phrases. Pure submodule, contracted, chain morphism, ring extension, base change, universal property, Prüfer domain, flat 
(when viewed as an inclusion of $R$-modules), then $R\left[\left\{X_{i}\right\}\right] \hookrightarrow S\left[\left\{X_{i}\right\}\right]$ is contracted for any (possibly infinite) set $\left\{X_{i}\right\}$ of commuting algebraically independent indeterminates over $S$.

It is convenient to summarize the last-mentioned result by saying that any pure ring extension is "universally contracted". This naturally raises the question whether the converse holds. In other words, if a ring extension $R \hookrightarrow S$ is universally contracted, must it be pure? According to [4, page 272], the answer is "yes", and a proof appears in an unpublished manuscript of Enochs [11]. In fact, to conclude "pure", one need only assume the "contracted" property for extensions of polynomial rings that involve finite sets $\left\{X_{i}\right\}$ of indeterminates. In order to make this result more widely available, we provide a proof of it in Proposition 2.

In view of the result of Enochs, it seems reasonable to ask if there are other naturally occurring ring-theoretic properties whose universalizations are equivalent to purity. In that regard, recall the following definition from [8, page 145]. A ring homomorphism $f: R \rightarrow S$ is called a chain morphism if every (possibly infinite) chain $\left\{P_{i}\right\}$ of prime ideals of $R$ can be "covered" by a corresponding chain $\left\{Q_{i}\right\}$ of prime ideals of $S$, in the sense that $f^{-1}\left(Q_{i}\right)=P_{i}$ for each $i$. The most familiar example of a chain morphism is provided by any integral ring extension $R \hookrightarrow S$; this generalization of the classical Goingup Theorem was proved in $[6$, Remark (d)]. By combining a result of Brenner [3, Theorem $3]$ with the model-theoretic analysis of templates by Bergman in [1], it was shown in [10, Proposition 2.5 (b)] that if $f: R \hookrightarrow S$ is contracted, then $f$ is a chain morphism. In view of Enochs' result, it therefore follows that if a ring extension $f: R \hookrightarrow S$ is universally contracted (that is, pure), then $f$ is a universally chain morphism. (Recall from [8] that a ring homomorphism $R \rightarrow S$ is said to be a universally chain morphism if $T \rightarrow T \otimes_{R} S$ is a chain morphism for each ring homomorphism $R \rightarrow T$.) The question naturally arises whether the converse holds. In other words, if a ring extension $f: R \hookrightarrow S$ is a universally chain morphism, must $f$ be pure? Sadly, we show in Example 3 that the construction of Lady gives a ring extension $f: R \hookrightarrow S$ which is a universally chain morphism but not pure. Nevertheless, there is a partial converse, as Theorem 4 establishes the following companion for [12, Corollary 4]: if $R \subseteq S$ are rings such that $R$ is a Prüfer domain, then $f: R \hookrightarrow S$ is pure if and only if $f$ is a universally chain morphism. Finally, Proposition 5 establishes that many of the concepts considered earlier become equivalent in the context of a flat ring extension $f: R \hookrightarrow S$.

As in [15, page 28], we let LO and GD denote the lying-over and going-down properties, respectively, of ring extensions (more generally, of ring homomorphisms). Any unexplained material is standard, as in [15].

The author wishes to thank Andrew Hetzel for some stimulating correspondence.

\section{Results}

We begin by collecting many useful facts. First, recall (cf. [14], [7]) that a ring homomorphism $f: R \rightarrow S$ is said to satisfy universally going-down if $T \rightarrow T \otimes_{R} S$ satisfies GD for each ring homomorphism $R \rightarrow T$, equivalently (by [7, Corollary 2.3]) 
if and only if $R\left[X_{1}, \ldots, X_{n}\right] \rightarrow S\left[X_{1}, \ldots, X_{n}\right]$ (the map induced by $f$ ) satisfies GD for each finite set $\left\{X_{1}, \ldots, X_{n}\right\}$ of commuting algebraically independent indeterminates over $S$.

\section{Lemma 1.}

(a) If $f: R \rightarrow S$ is a contracted ring homomorphism, then $f$ is an injection.

(b) If $f: R \rightarrow S$ is a ring homomorphism making $S$ a flat $R$-module and $\iota: M \hookrightarrow N$ is a pure inclusion of $R$-modules, then $1 \otimes \iota: S \otimes_{R} M \rightarrow S \otimes_{R} N$ is a pure inclusion of $R$-modules.

(c) "Faithfully flat" is a universal property of ring homomorphisms. More precisely, if $f: R \rightarrow S$ is a ring homomorphism and $M$ is a faithfully flat $R$-module, then $S \otimes_{R} M$ is a faithfully flat $S$-module.

(d) If $f: R \rightarrow S$ is a ring homomorphism making $S$ is a faithfully flat $R$-module, then $f$ is contracted.

(e) If $R \subseteq S$ are rings such that $S$ is a faithfully flat $R$-module, then $R \hookrightarrow S$ is pure (as an inclusion of $R$-modules).

(f) If a ring homomorphism $f: R \rightarrow S$ satisfies $L O$ and universally going-down, then $f$ is a universally chain morphism.

(g) If $f: R \rightarrow S$ is a contracted ring homomorphism and $I$ is an ideal of $R$, then the induced injective ring homomorphism $\bar{f}: R / I \rightarrow S / I S$ is contracted.

Proof. For (a), we model the proof of [2, Proposition 8 (iii), page 32], as follows. Use the fact that $f$ is contracted to get that the ideal $I:=0$ satisfies $0=I=f^{-1}(I S)=$ $f^{-1}(0 S)=f^{-1}(0)=\operatorname{ker}(f)$. For (b), note first, by flatness, that the canonical map of $S$-modules $1 \otimes \iota: S \otimes_{R} M \rightarrow S \otimes_{R} N$ is an injection and, hence, can be viewed as an inclusion. Next, it suffices to note that if $E$ is an $S$-module, then the canonical map $\left(S \otimes_{R} M\right) \otimes_{S} E \rightarrow\left(S \otimes_{R} N\right) \otimes_{S} E$ can be identified with the canonical (pure) $R$-module map $M \otimes_{R} E \rightarrow N \otimes_{R} E$. As for (c), note that this is just a restatement of [2, Proposition 5 , page 31] for the case of commutative rings. Similarly, (d) is just a restatement of [2, Proposition 8 (ii), page 32]. As for (e), one needs to prove that if $E$ is an $R$-module, then the canonical map $E \cong E \otimes_{R} R \rightarrow E \otimes_{R} S$ is an injection, and this is just a restatement of $[2$, Proposition 8 (i), page 32]..

(f) Recall that LO is a universal property; that is, LO is preserved by arbitrary base changes. To see this, apply [14, Corollaire 3.2.7.1 (i), page 235], as in the proof of [14, Proposition 3.6.1 (ii), page 244]. Accordingly, to prove (f), it will suffice to show that if a ring homomorphism $f: R \rightarrow S$ satisfies LO and GD, then $f$ is a chain morphism. To that end, it suffices to combine [8, Proposition 3.2 (a)] and [9, Theorem 2.2].

(g) Since $f$ is contracted, $\operatorname{ker}(\bar{f})=(I S \cap R) / I=0$, and so $\bar{f}$ is, indeed, an injection. It now suffices to show that if $J$ is an ideal of $R$ such that $J \supseteq I$, then the ideal $\bar{J}:=J / I S$ of $\bar{S}:=S / I S$ satisfies $\bar{f}^{-1}(\bar{J} \bar{S})=\bar{J}$. This, in turn, can be shown by a easy calculation using the fact that $f^{-1}(J S)=J$ as a consequence of $f$ being contracted (and bearing in mind that $\bar{J} \bar{S}=(J S) /(I S))$. 
Proposition 2 provides a proof of the result of Enochs [11] that a ring extension is pure if (and only if) it is universally contracted. When I requested a copy of [11] a few years ago, Enochs kindly provided what he had at hand, namely, an incomplete copy of the manuscript which unfortunately lacked the pages that apparently contained the formal statement and proof of the result in question. Although the authors of [4] presumably saw a complete copy of [11] some 25 years ago, Brewer is now deceased and Costa has left academia, and so I can only hope that the proof given below is in the spirit of Enochs' original proof.

Proposition 2. (Enochs) Let $f: R \rightarrow S$ be a ring homomorphism. Then the following conditions are equivalent:

(1) $f$ is universally contracted, in the sense that for any (possibly infinite) set $\left\{X_{i}\right\}$ of commuting algebraically independent indeterminates over $S$, $f$ induces a map $R\left[\left\{X_{i}\right\}\right] \rightarrow S\left[\left\{X_{i}\right\}\right]$ which is contracted;

(2) For any finite set $\left\{X_{1}, \ldots, X_{n}\right\}$ of commuting algebraically independent indeterminates over $S, f$ induces a map $R\left[X_{1}, \ldots, X_{n}\right] \rightarrow S\left[X_{1}, \ldots, X_{n}\right]$ which is contracted;

(3) $f$ is pure (when viewed as an inclusion map of $R$-modules).

Proof. A proof that $(3) \Rightarrow(1)$ was included in the first paragraph of the Introduction. Moreover, $(1) \Rightarrow(2)$ trivially. It remains to prove that $(2) \Rightarrow(3)$. Assume (2). It follows easily from Lemma 1 (a) that $f$ is an injection. We will prove that the canonical homomorphism $g: E \cong R \otimes_{R} E \rightarrow S \otimes_{R} E$ is an injection for each $R$-module $E$. It is well known that $E$ can be assumed to be a finitely presented $R$-module (cf. [17, page 703 , lines 7-10]), say identified with $R\left[X_{1}, \ldots, X_{n}\right] / I$ for some finite set $\left\{X_{1}, \ldots, X_{n}\right\}$ as in the statement of (2) and some finitely generated ideal $I$ of $R\left[X_{1}, \ldots, X_{n}\right]$. For convenience, set $A:=R\left[X_{1}, \ldots, X_{n}\right]$ and $B:=S\left[X_{1}, \ldots, X_{n}\right]$. Then $g$ can be identified with

$$
h: A / I \rightarrow S \otimes_{R} A / I \cong\left(S \otimes_{R} A\right) \otimes_{A} A / I \cong B \otimes_{A} A / I \cong B / I B
$$

It suffices to prove that $h$ is an injection. Since we have assumed that $f$ induces a contracted ring homomorphism $A \rightarrow B$, Lemma 1 (g) shows that $h$ is contracted. Hence, by Lemma 1 (a), $h$ is an injection, as desired.

We next revisit the example suggested by E. L. Lady in [4, Example 1], pointing out some of its properties. Propositions 4 and 5 will go on to examine such properties in some different contexts.

Example 3. In [4, Example 1], there is a construction of a ring extension $R \subseteq S$ such that $R$ is an Artinian ring and the inclusion map $f: R \hookrightarrow S$ is contracted but not pure (as an inclusion of $R$-modules). Any such data have the following additional features:

(a) $f$ satisfies LO and universally going-down, and $f$ is a universally chain morphism.

(b) $S$ is not an $R$-flat module (via the structure induced by $f$ ). 
Proof. (a) We use the Artinian property of $R$ only to conclude that $R$ has Krull dimension 0, for then $f$ satisfies both LO (by [15, Exercise 1, page 41]) and universally going-down (by [7, Proposition 3.3]). Therefore, by Lemma 1 (f), $f$ is also a universally chain morphism.

(b) If the assertion fails, then the "flat" and (from (a)) "LO" properties combine to ensure that $S$ is faithfully flat over $R$ [2, Proposition 9, page 33], whence $f$ is pure by Lemma 1 (e), the desired contradiction.

We pause to note that one reason for the inclusion of part (b) of Example 3 is that any ring homomorphism that imparts a flat module structure must satisfy universally going-down [14, Corollaire 3.9.4 (ii)]. The "flat" context will be examined more fully in Proposition 5.

As explained in the third paragraph of the Introduction, it follows by combining [10, Proposition 2.5 (b)] with Proposition 2 that if $R \subseteq S$ are rings such that $R \hookrightarrow S$ is pure (as an inclusion of $R$-modules), then $R \hookrightarrow S$ is a universally chain morphism. Example 3 shows that the converse does not hold in general. However, we next show that the converse does hold if $R$ is a Prüfer domain.

First, recall from [16], [8] that a ring homomorphism $f: R \rightarrow S$ is called subtrusive (also known as a 2-chain morphism) if, for each inclusion $P_{1} \subseteq P_{2}$ of prime ideals of $R$, there exist prime ideals $Q_{1} \subseteq Q_{2}$ of $S$ such that $f^{-1}\left(Q_{i}\right)=P_{i}$ for $i=1,2$. Of course, a "universally 2-chain morphism" is a ring homomorphism $R \rightarrow S$ such that $T \rightarrow T \otimes_{R} S$ is a 2-chain morphism for each ring homomorphism $R \rightarrow T$.

Proposition 4. Let $R \subseteq S$ be rings such that $R$ is a Prüfer domain, and let $f$ denote the inclusion map $f: R \hookrightarrow S$. Then the following conditions are equivalent:

(1) $f$ is contracted;

(2) $f$ is a universally chain morphism;

(3) $f$ is pure (as an inclusion of $R$-modules).

Proof. $(3) \Rightarrow(1)$ : As noted in the Introduction, this implication was established by Gilmer and Mott [12, page 752] in general (that is, regardless of whether the ring $R$ is a Prüfer domain). It has also been noted by other workers (cf. [4, page 271]).

$(1) \Rightarrow(3)$ : This implication was established by Gilmer and Mott [12, Corollary 4] (using and needing the hypothesis that $R$ is a Prüfer domain).

$(3) \Rightarrow(2)$ : Assume (3). By [16, page 538], $f$ is universally subtrusive, that is, a universally 2 -chain morphism. However, $[8$, Theorem 3.26] established that the universally 2-chain morphisms are the same as the universally chain morphisms, and so (2) follows.

$(2) \Rightarrow(3)$ : Assume (2). We must show that if $E$ is any $R$-module, then the canonical map $E \cong R \otimes_{R} E \rightarrow S \otimes_{R} E$ is an injection; equivalently, for each maximal ideal $M$ of $R$, the canonical map

$$
E_{M}:=E_{R \backslash M} \cong E \otimes_{R} R_{M} \rightarrow\left(S \otimes_{R} E\right) \otimes_{R} R_{M} \cong S_{M} \otimes_{R_{M}} E_{M}
$$


is an injection. (For lack of a convenient reference, we have included the above proof that purity is a local property, a fact that is surely known.) Thus, we may replace $R$ with the valuation domain $R_{M}$ (and $f$ with $R_{M} \hookrightarrow S_{M}$ ). Hence, we can obtain (3) by applying [16, Proposition 16], a result that includes the following statement: if $h: V \hookrightarrow S$ is a (universally) 2-chain morphism such that $V$ is a valuation domain, then $h$ is pure.

Note that the equivalence $(1) \Leftrightarrow(3)$ in Proposition 4 was obtained by Gilmer and Mott in [12, Remark 6]. We wish also to emphasize that if the $\operatorname{ring} R$ is not assumed to be a Prüfer domain, then the conditions in Proposition 4 satisfy $(3) \Rightarrow(1) \Rightarrow(2) \nRightarrow(3)$ in general (by [12, page 752], [10, Proposition 2.5 (b)] and Example 3).

Proposition 5. Let $R \subseteq S$ be rings such that $S$ is a flat $R$-module. For the inclusion map $f: R \hookrightarrow S$, the following conditions are equivalent:

(1) $f$ is contracted;

(2) $f$ is universally contracted;

(3) $f$ satisfies $L O$;

(4) $f$ is faithfully flat (that is, $S$ is faithfully flat as an $R$-module);

(5) $f$ is pure (as an inclusion of $R$-modules);

(6) $f$ is a chain morphism;

(7) $f$ is a universally chain morphism.

Proof. By [2, Proposition 9, page 33], (1) $\Leftrightarrow(3) \Leftrightarrow(4)$. (Note that the " $\Rightarrow$ " part of the first " $\Leftrightarrow$ " also uses the fact that $f$ being flat implies that $f$ satisfies (universally) GD [14, Corollaire 3.9.4 (ii), page 254].) Next, to prove that (4) $\Rightarrow(2)$, use Lemma 1 (c) to reduce the task to showing that (4) implies (1), and then apply Lemma 1 (d). As (2) $\Rightarrow$ (1) trivially, we have shown the equivalence of (1), (2), (3), and (4). Moreover, (4) $\Leftrightarrow(5)$ by Proposition 2. As the implications $(7) \Rightarrow(6) \Rightarrow(3)$ are trivial, it therefore suffices to prove that $(3) \Rightarrow(7)$. Thus, an application of Lemma 1 (f) completes the proof.

Remark 6 (a) The assumption of flatness is needed in proving several of the implications in Proposition 5. We content ourselves by mentioning only one such here, namely, that in general, $(3) \nRightarrow(4)$. In particular, if a ring extension $R \subseteq S$ satisfies LO, $S$ need not be a flat $R$-module. For the easiest example showing this, note that any proper integral overring inside the total quotient ring must satisfy LO (cf. [15, Theorem 44]) but cannot be flat [13, Proposition 12].

(b) Lemma 1 (f) played a decisive role in some of the above proofs. We would like to point out that the "universally going-down" hypothesis cannot be deleted from Lemma 1 (f). In other words, a ring extension $f: R \hookrightarrow S$ that satisfies LO need not be a universally chain morphism. Indeed, in view of the example of a 1-chain morphism that is not a 2-chain morphism in [10, Example 2.2], $f$ need not even be a 2-chain morphism.

(c) A familiar argument using Zorn's Lemma can be used to show that any contracted ring homomorphism satisfies LO (cf. [15, Theorem 1]). This fact can be used to prove a 
result that is motivated by Example 3 and Propositions 4 and 5 . The result asserts that if $f: R \rightarrow S$ is a contracted ring homomorphism where $R$ is a ring of Krull dimension 0 , then $f$ is a universally chain morphism. For a proof, note that $f$ satisfies universally going-down (by [7, Proposition 3.3]), and so an application of Lemma 1 (f) yields the assertion.

(d) We close by recording the following improvement of Lemma 1 (f). If a ring homomorphism $f: R \rightarrow S$ satisfies universally going-down, then: $f$ satisfies LO $\Leftrightarrow f$ is a chain morphism $\Leftrightarrow f$ is universally a chain morphism. In view of Lemma 1 (f), the proof reduces to the observation that any chain morphism satisfies LO.

\section{References}

[1] G. M. Bergman, Arrays of prime ideals in commutative rings, J. Algebra 261 (2003), 389410.

[2] N. Bourbaki, Commutative Algebra, Addison-Wesley, Reading, Mass., 1972.

[3] H. Brenner, Lifting chains of prime ideals, J. Pure Appl. Algebra 179 (2003), 1-5.

[4] J. W. Brewer and D. L. Costa, Contracted ideals and purity for ring extensions, Proc. Amer. Math. Soc. 53 (1975), 271-276.

[5] P. M. Cohn, On the free product of associative rings, Math. Zeit. 71 (1959), 380-398.

[6] D. E. Dobbs, A going-up theorem for arbitrary chains of prime ideals, Comm. Algebra 27 (1999), 3887-3894. Corrigendum 28 (2000), 1653-1654.

[7] D. E. Dobbs and M. Fontana, Universally going-down homomorphisms of commutative rings, J. Algebra 90 (1984), 410-429.

[8] D. E. Dobbs, M. Fontana and G. Picavet, Generalized going-down homomorphisms of commutative rings, pp.143-163 in Commutative Ring Theory and Applications, Lecture Notes Pure Appl. Math. 231, Dekker, New York, 2002.

[9] D. E. Dobbs and A. J. Hetzel, Going-dowm implies generalized going-down, Rocky Mountain J. Math. 35 (2005), 479-484.

[10] D. E. Dobbs and A. J. Hetzel, On chain morphisms of commutative rings, Rend. Circ. Mat. Palermo 53 (2004), 71-84.

[11] E. Enochs, On absolutely pure modules, unpublished manuscript.

[12] R. W. Gilmer and J. L. Mott, Some results on contracted ideals, Duke Math. J. 37 (1970), 751-767.

[13] M. Griffin, Prüfer rings with zero divisors, J. Reine Angew. Math. 240 (1970), 55-67.

[14] A. Grothendieck and J. Dieudonné, Eléments de Géométrie Algébrique, Springer-Verlag, Berlin, 1971.

[15] I. Kaplansky, Commutative Rings, rev. ed., Univ. Chicago Press, Chicago, 1974.

[16] G. Picavet, Submersion et descente, J. Algebra 103 (1986), 527-591.

[17] R. B. Warfield, Jr., Purity and algebraic compactness for modules, Pacific J. Math. 28 (1969), 699-719.

Department of Mathematics, University of Tennessee, Knoxville, Tennessee 37996-1300, U.S.A.

E-mail: dobbs@math.utk.edu 